Check for updates

The BMJ

Cite this as: BMJ 2021;372:n309 http://dx.doi.org/10.1136/bmj.n309 Published: 02 February 2021

\title{
Covid-19: Russian vaccine efficacy is $91.6 \%$, show phase III trial results
}

\section{Elisabeth Mahase}

The covid-19 vaccine developed by the Gamaleya Research Institute, part of the Russian Ministry of Health, has an efficacy of $91.6 \%$, according to interim results from a phase III trial. ${ }^{1}$

The findings, published in the Lancet, showed that the two dose regime of the viral vector vaccine was generally well tolerated with no associated serious adverse events and similar efficacy in those aged over and under 60.

The Gam-COVID-Vac, also known as Sputnik V, was tested at 25 hospitals and polyclinics in Moscow between 7 September and 24 November 2020. Just under 15000 volunteers received the vaccine (of whom 1611 were aged 60 or over) and 4902 received the placebo (vaccine buffer composition).

The primary outcome was the proportion of participants with polymerase chain reaction confirmed covid-19 from day 21 after receiving the first dose. From 21 days after the first dose of vaccine (the day of dose two), the trial reported 16 covid-19 cases in the vaccine group (0.1\%) and 62 cases (1.3\%) in the placebo group. However, volunteers were only tested for covid-19 when they self-reported symptoms. The efficacy data therefore only includes symptomatic cases.

This equated to an overall efficacy of $91.6 \%$. When broken down by age, the study reported efficacy as $91.8 \%$ in those 60 and over, $92.7 \%$ in those 51 to 60 , $91.3 \%$ in those 41 to $50,90.0 \%$ for those 31 to 40 , and $91.9 \%$ for those aged 18 to 30 .

The study also found that 14 days after the first dose, vaccine efficacy was $87.6 \%$. Any time after the first dose, the efficacy was $73.1 \%$. Researchers are now investigating a single dose regimen of the vaccine.

The secondary outcomes included severity of illness and incidence or severity of adverse events. There were no moderate or severe cases of covid-19 in the vaccine group, but 20 reported in the placebo group. Most reported adverse events were mild, including flu like symptoms, pain at injection site, and weakness or low energy.

The vaccine uses similar technology to the Oxford AstraZeneca vaccine, however it follows a prime and then boost regime. The two shots use different vectors - the first $\mathrm{rAd}_{2} 6$ and the second $\mathrm{rAd}_{5}-$ but both carry the gene for the full SARS-CoV-2 spike protein.

Alexander Edwards, associate professor in biomedical technology at the University of Reading, said that the vaccine design "aims to drive higher immune responses to the target spike by using two slightly different jabs-the only shared element of the inoculation being the covid-19 spike. This is thought to be beneficial for adenovirus based vaccines as otherwise, if you have two identical vaccine doses, your immune system can be so efficient that it eliminates the second dose so quickly that your immunity to spike isn't boosted as much.”

He added, "While heterologous prime boost has been discussed and explored experimentally for the past 20 years, this could be the large scale human trial that finally proves how well the approach can work to prevent a widespread human disease. Further clinical trials are urgently needed to understand the best way to combine different vaccine doses for maximum protection, especially if regular vaccine programmes similar to the annual influenza program become important."

The Gam-COVID-Vac trial is ongoing and aims to recruit a total of 40 ooo participants.

Logunov DY, Dolzhikova IV, Shcheblyakov DV, et al. Safety and efficacy of an rAd26 and rAd5 vector-based heterologous prime-boost covid-19 vaccine: an interim analysis of a randomised controlled phase 3 trial in Russia. Lancet. 2021. www.thelancet.com/journals/lancet/article/PIIS01406736(21)00234-8/fulltext 